\title{
Effect of Electroacupuncture on Reproductive Disorders and Insulin Resistance in a Murine Polycystic Ovary Syndrome Model
}

\author{
Chang Ji, ${ }^{1}$ Wanling Xu, ${ }^{1}$ Zhiqing Zhang, ${ }^{1,2}$ Shuai Cui, ${ }^{1,3}$ and Wei Yi ${ }^{1}{ }^{1}$ \\ ${ }^{1}$ South China Research Center for Acupuncture and Moxibustion, Medical College of Acu-Moxi and Rehabilitation, \\ University of Chinese Medicine, Guangzhou 510006, China \\ ${ }^{2}$ Acupuncture Research Team, The Second Affiliated Hospital of Guangzhou University of Chinese Medicine, Guangzhou, China \\ ${ }^{3}$ Research Institute of Acupuncture and Meridian, Anhui University of Chinese Medicine, Hefei, China \\ Correspondence should be addressed to Wei Yi; yw8013@gzucm.edu.cn
}

Received 30 March 2021; Revised 2 September 2021; Accepted 15 September 2021; Published 26 December 2021

Academic Editor: Jeong June Choi

Copyright (c) 2021 Chang Ji et al. This is an open access article distributed under the Creative Commons Attribution License, which permits unrestricted use, distribution, and reproduction in any medium, provided the original work is properly cited.

\begin{abstract}
Polycystic ovarian syndrome (PCOS) is a common, complex, and heterogeneous endocrine and metabolic disorder. There is no standardized treatment, and it therefore requires individualized therapies according to the symptoms and pathogenesis of each patient. The present study aimed to determine the effect of electroacupuncture at the acupoints Zusanli (ST36), Sanyinjiao (SP6), and Neiguan (PC6) on reproductive disorders and insulin resistance in a murine model of PCOS induced by dehydroepiandrosterone (DHEA). Vaginal smear analysis was used to determine mice estrous cycle; intraperitoneal glucose and insulin tolerance tests were adopted to analyze metabolic characteristics; enzyme-linked immunosorbent assay was used to measure hormone levels; gene expression was quantified with real-time PCR; hematoxylin and eosin staining was used to observe ovarian morphology. We observed disordered estrous cycle, polycystic ovarian morphology, and higher levels of homeostasis model assessment-insulin resistance (HOMA-IR) and testosterone (T), indicating successful modeling of PCOS. DHEA increased levels of estrogen $\left(\mathrm{E}_{2}\right)$, progesterone $(\mathrm{P})$, testosterone $(\mathrm{T})$, luteinizing hormone $(\mathrm{LH})$, and follicle-stimulating hormone (FSH), and EA treatment restored them to levels seen in the control group. EA reduced the days in estrus caused by DHEA, improved the abnormal sex hormone receptor genes, and attenuated the DHEA-induced histomorphological changes in mouse ovaries. The average expressions of the androgen receptor (AR), estrogen receptor (ER), luteinizing hormone receptor (LHR), and folliclestimulating hormone receptor (FSHR) genes in the ovary greatly increased after DHEA treatment and significantly decreased in the DHEA + EA group. After EA treatment, the cystic follicle (CF) number was reduced and corpora lutea (CL) increased in the DHEA + EA group compared to the DHEA group. EA improved glucose intolerance and insulin intolerance. Statistical analysis of intraperitoneal glucose tolerance test-area under curve (IPGTT-AUC) glucose levels revealed a significant decrease in DHEA group mice compared to the control and DHEA + EA groups. EA was found to restore fasting blood glucose, fasting serum insulin, and HOMA-IR. In summary, our study suggests that EA has a remarkable effect in the DHEA-induced murine PCOS model. Management of EA could improve estrous cycle, hormonal disorders, abnormal sex hormone receptors in ovaries, ovary morphology, and insulin resistance in PCOS mice.
\end{abstract}

\section{Introduction}

Polycystic ovarian syndrome (PCOS) is a common but complex and heterogeneous endocrine and metabolic disorder characterized by anovulation or oligoovulation, hyperandrogenism, and polycystic ovarian morphology [1-4]. Approximately 5-10\% of reproductive-age women develop PCOS, as a result of either genetics or lifestyle conditions such as being in a dark environment for long periods of time, overeating, or not getting enough exercise [5]. Primary clinical manifestations of PCOS include ovulatory dysfunction, infertility, hirsutism, acne, and obesity [6]. Women with PCOS have more frequent pulses of gonadotropin-releasing hormone $(\mathrm{GnRH})$ and luteinizing hormone (LH), as well as an increased LH/follicle-stimulating hormone (FSH) ratio. At the hypothalamic-pituitary 
level, progesterone is not able to regulate the secretion of GnRH as it does in women without PCOS [7]. The ovarian theca cells secrete higher levels of androgen because of the increased LH.

According to histological analyses, the ovaries of PCOS patients have numerous antral follicles; however, the etiology needs further research. Reactive oxygen species (ROS) induced by PCOS could cause ovarian damage, and some reports have shown that antioxidants have a positive effect on ovaries $[8,9]$. Reports show that PCOS is closely associated with hypothalamus-pituitary-ovarian axis dysfunction, hyperandrogenism, insulin resistance (IR), hyperinsulinemia, and type II diabetes [10, 11]. Although IR is a common pathologic manifestation of PCOS (seen in $\sim 85 \%$ of PCOS patients, especially those who are overweight), it is not one of the diagnostic criteria for PCOS. Research has shown that IR and obesity have a close linkage with PCOS; clinical manifestations of PCOS are more apparent in patients with IR and obesity [12-14]. The relationship between PCOS and IR is still unknown, and further research is required. There are currently no effective cures for PCOS. The development of new therapies with increased effectiveness and fewer side effects remains an urgent need.

There have been reports that both pharmacological and nonpharmacological therapies for PCOS may cause side effects such as cardiovascular disease and multifetation $[15,16]$. A great number of clinical trials and animal studies have reported that electroacupuncture (EA) treatment could effectively relieve PCOS symptoms in humans. Jedel et al. found that EA had a better effect in the treatment of hyperandrogenism and oligo/amenorrhea [17] compared to physical exercise. Yu et al. indicated that EA could improve obesity-related indexes and insulin sensitivity of obese PCOS patients [18]. Through a randomized controlled trial, Cao et al. concluded that Tung's acupuncture could regulate menstruation and control weight [19]. Compared with the attention and time involved in meeting with a therapist, 10-13 weeks of acupuncture treatment showed more improvement of ovulation frequency in lean/overweight women with PCOS, suggesting that acupuncture is more effective than placebo [20]. These data illustrate that EA treatment can be used to treat oligo/amenorrhea, obesity, and IR in women with PCOS. However, some randomized clinical trials indicate that acupuncture has no apparent effect on PCOS [21]. In animal experiments, a previous study showed that the pathological symptoms of PCOS in rats, such as serum hormone levels and insulin resistance, were improved after EA treatment [22]. Cui et al. indicated that PCOS rats restored estrous cyclicity, increased numbers of preovulatory follicles and corpora lutea, and decreased body weight after four weeks of EA treatment [23]. In total, the effects of acupuncture in humans and animals with PCOS are controversial, and more experiments are needed to provide clarification.

Previous research has demonstrated that DHEA-induced PCOS models have various representative characteristics such as estrous cycle disorder, impaired sensitivity to glucose and insulin, disruption of hormones, and change in ovarian morphology [24-27]. In the present study, we established a PCOS mouse model by DHEA injection and studied the effects of EA on the model.

\section{Materials and Methods}

2.1. Animal Model and Experimental Design. Thirty 21-dayold female C57BL/6J mice were purchased from the Experimental Animal Center of Guangzhou University of Chinese Medicine (SCXK (Yue) 2018-0034). All animals were housed in South China Research Center for Acupuncture and Moxibustion $\left(24^{\circ} \mathrm{C} \pm 1^{\circ} \mathrm{C}, 12 / 12 \mathrm{~h}\right.$ light/dark cycle), with sufficient food and water freely available. All animal studies described in this article were approved by the Ethical Committee of Guangzhou University of Chinese Medicine.

On day 25 after birth, the mice were randomly placed into two groups: the control group $(n=12)$ and the DHEA group $(n=18)$. The DHEA group was given DHEA subcutaneously across their back $(6 \mathrm{mg} / 100 \mathrm{~g}, 100 \mu \mathrm{l} /$ mouse in sesame oil with $10 \%$ of $95 \%$ ethanol, [Sigma-Aldrich, St. Louis, MO, USA]) for 20 consecutive days. The control group mice were injected with $90 \mu \mathrm{l}$ sesame oil and $10 \mu \mathrm{l} 95 \%$ ethanol daily for 20 consecutive days. Estrous cycle was monitored daily from day 10 after DHEA treatment until the end of the experiments. After 20 days of treatment, six mice were selected at random from each group to confirm the PCOS model, and the DHEA group was randomly divided into two groups: the DHEA control group $(n=6)$ and the DHEA + EA group $(n=6)$. The DHEA + EA group received EA stimulation at the acupoints Zusanli (ST36), Sanyinjiao (SP6), and Neiguan (PC6). Mice were stimulated with density waves from a Hwato SDZ-III machine at a frequency of $2 \mathrm{~Hz}$ and an intensity of $1 \mathrm{~mA}$ for $20 \mathrm{~min}$ per day for four weeks. When the EA treatment was finished, the mice were fasted overnight, anesthetized, and then sacrificed. Serum samples and left ovaries were collected and stored at $-80^{\circ} \mathrm{C}$ for ELISA and real-time PCR. The left ovaries were dissected for hematoxylin and eosin staining.

2.2. Estrous Cycle Detection. Vaginal smear analysis was performed daily at 9 am from 10 days after DHEA treatment. Vaginal cells were collected via saline lavage and dropped onto a glass slide. After air-drying, they were stained with $0.1 \%$ methylene blue (Solarbio, China). Estrous stages were determined based on the predominant cell type as described previously $[24,28]$.

2.3. Intraperitoneal Glucose Tolerance Test and Insulin Tolerance Test. After 20 days of DHEA treatment, the mice fasted for $12 \mathrm{~h}$ before the experiment. Blood was extracted from the tail vein following disinfection of the tail with $75 \%$ ethanol. After measuring the fasting glucose levels at 9 am by ACCU-CHEK Performa (YZB/GER 5513-2014), the mice were given D-glucose (Sigma-Aldrich, $2 \mathrm{~g} / \mathrm{kg}$ body weight as a $50 \%$ glucose stock) through intraperitoneal injection. Blood glucose was then measured at 30,60, 90, and $120 \mathrm{~min}$.

Three days after the intraperitoneal glucose tolerance test (IPGTT) experiment, mice were fasted for $6 \mathrm{~h}$ before 
intraperitoneal injection with insulin (1 IU/kg body weight, Humalog Mix25, China). Tail blood sampling was performed at 9 am and then at 30,60, 90, and $120 \mathrm{~min}$ after intraperitoneal injection.

2.4. Hormone Measurements. At the end of the EA treatment, the mice were anesthetized and blood samples were collected from the orbit. After standing at room temperature for $2 \mathrm{~h}$, blood samples were centrifuged at $2500 \mathrm{rpm}$ for $15 \mathrm{~min}$. The upper serum was extracted and stored at $-80^{\circ} \mathrm{C}$. Serum estrogen, progesterone, testosterone, follicle-stimulating hormone, luteinizing hormone, and insulin were determined with mice kits (Elabscience, China). The procedures were carried out in accordance with the manufacturer's instructions.

2.5. Real-Time PCR. Ovaries were collected and stored immediately at $-80^{\circ} \mathrm{C}$. Total RNA was extracted from the ovaries using TRIzol reagent (Life Technologies) according to the manufacturer's instructions. cDNA was generated with the PrimeScript reverse transcriptase reagent kit (Perfect Real Time; TaKaRa, Shiga, Japan). Real-time PCR (RT-PCR) was performed using TB Green Premix Ex Taq II (Tli RNase H Plus, TaKaRa). The program used for RT-PCR was as follows: $95^{\circ} \mathrm{C}$ for $30 \mathrm{~s} ; 40$ cycles of $95^{\circ} \mathrm{C}$ for $5 \mathrm{~s}$ and $60^{\circ} \mathrm{C}$ for $31 \mathrm{~s} ; 95^{\circ} \mathrm{C}$ for $15 \mathrm{~s}$; $60^{\circ} \mathrm{C}$ for $1 \mathrm{~min} ; 95^{\circ} \mathrm{C}$ for $15 \mathrm{~s}$; and $60^{\circ} \mathrm{C}$ for $15 \mathrm{~s}$. Relative gene expression was calculated using the $2^{-\Delta \Delta C T}$ method. The primer sequences are AR: forward: TAAAGACATTTTG AACGAGGCC, reverse: GTCAGATATGGTTGAATTGCCC; ER: forward: CTACTACCTGGAGAACGAGC, reverse: GCG TCGATTGTCAGAATTAGAC; PR: forward: TAGTCTC GCCTATACCGATCTC, reverse: CTTCCCTATGAGTG GCTTCTAC; GNRHR: forward: ATTCTCAGCATTGT CTTTGCAG, reverse: GCA-TTGCAGATTAGCATGATGA; GAPDH: forward: GCACCACCAACTGCTTAG, reverse: CAGTGATGGCATGGACTG.

2.6. H\&E Staining. The left ovaries were fixed with $4 \%$ paraformaldehyde at $4^{\circ} \mathrm{C}$ for $24 \mathrm{~h}$. After tissue dehydration and paraffin embedding, the ovaries were cut and sliced into $5 \mu \mathrm{m}$ serial sections with a tissue slicer (Thermo Scientific HM340 E, Germany). To get the most considerable sections of each ovary, one out of every five sections was selected. The sections were stained using the hematoxylin and eosin staining kit (Beyotime, China) following the manufacturer's guidelines. Corpora lutea were counted using an optical microscope (Nikon Eclipse E200, Japan).

2.7. Statistical Analysis. All statistical analyses were performed in GraphPad Prism (GraphPad Software Inc., San Diego, CA, USA). One-way analysis of variance (ANOVA) followed by Tukey's post hoc test was used to analyze differences among groups. $p<0.05$ was considered statistically significant.

\section{Results}

3.1. Confirmation of the PCOS Model. In order to evaluate the establishment of the PCOS model, the estrous cycle was monitored by vaginal smear examination during the experimental period. The control group mice had regular estrus as described previously $[24,28]$ : a significant number of nuclear epithelial cells appeared to be in the proestrus stage (Figure 1(a)); squamous epithelial cells accounted for the majority of cell types in the estrus stage (Figure 1(b)); nuclear epithelial cells, squamous epithelial cells, and leukocytes were observed in the metestrus stage (Figure 1(c)); and almost all cells were leukocytes in the diestrus stage (Figure 1(d)). The control mice had a regular estrus stage, while in the DHEA group almost all mice were in the estrus stage (Figures 1(e) and 1(f)), which was a typical pathological feature of PCOS. The number of days in the estrus stage increased significantly in the DHEA group (Figure $1(\mathrm{~g})$ ). HOMA-IR was calculated (HOMA-IR = FBG $(\mathrm{mmol} / \mathrm{L}) \times$ FINS $(\mathrm{mU} / \mathrm{L}) / 22.5)$ and compared between the control group and the DHEA group after DHEA injection (Figure 1(h)). HOMA-IR was significantly increased in PCOS mice. The DHEA group had higher serum $T$ levels than the control mice (Figure 1(i)). Normal ovaries had follicles at various developmental stages and several corpora lutea (CL) (Figure 1(j)), whereas ovaries of the DHEA group had several cystic follicles (CF) (Figure 1(k)).

Compared with the control group, the numbers of CL were significantly decreased (Figure 1(1)) and the numbers of CF were significantly increased (Figure $1(\mathrm{~m})$ ). These results indicated that induction of the PCOS model was successful.

3.2. EA Improves Estrous Cycle and Serum Hormone Levels. After EA treatment, we monitored the estrous cycles of the three groups (Figures 2(a)-2(c)). The days of estrus in the DHEA + EA group were significantly reduced compared with the DHEA group but did not recover to the levels of control mice (Figure 2(d)). The average estrogen level in the DHEA group increased by about 1.5 times after treatment with DHEA. EA significantly decreased estrogen levels, although they did not return to control levels (Figure 2(e)). However, DHEA increased progesterone, testosterone, $\mathrm{LH}$, and FSH levels by two to three times, and EA treatment restored them to the control group levels (Figures 2(f)-2(i)). Overall, treatment with EA improved estrous cycle and serum hormone levels.

3.3. EA Improves Abnormal Sex Hormone Receptors and Attenuates the Histomorphological Changes of Ovaries Induced by DHEA in Mice. The average expression of the AR gene in the DHEA group increased approximately 1.5 times after DHEA treatment. After EA treatment, androgen receptor (AR) gene expression was decreased to the control level (Figure 3(a)). The estrogen receptor (ER) gene in the DHEA group increased approximately three times after DHEA treatment. Following EA intervention, ER expression was comparable to the control group (Figure 3(b)). The average expression of the luteinizing hormone receptor (LHR) gene in the DHEA group increased about six times after DHEA treatment. EA treatment also decreased 


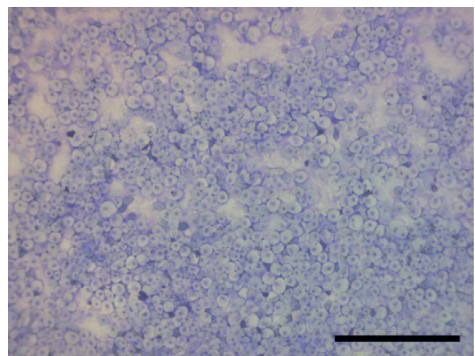

(a)

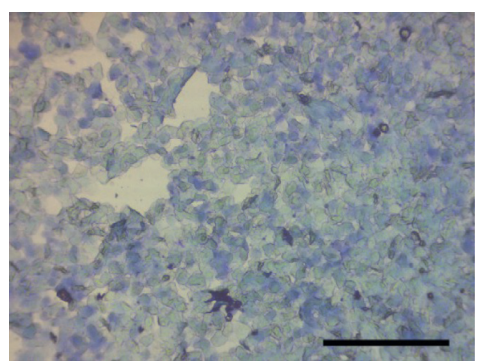

(b)

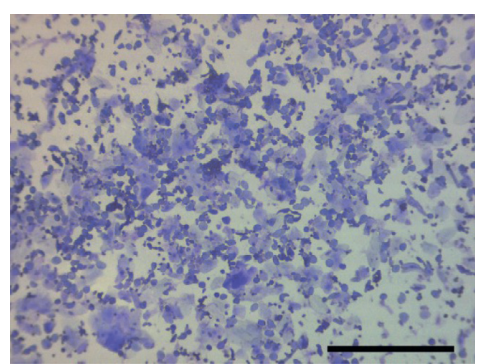

(c)

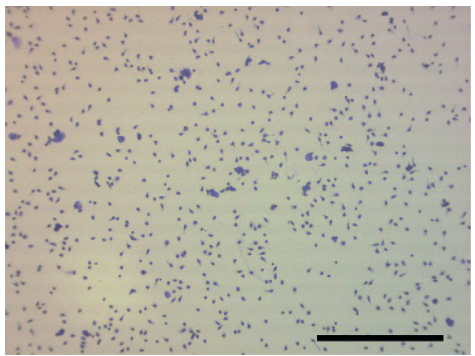

(d)
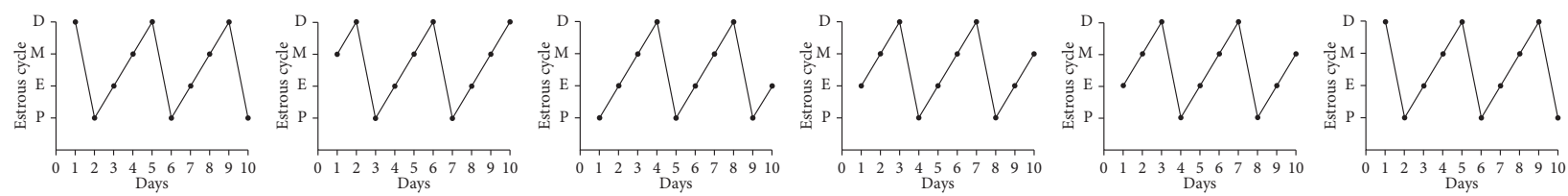

(e)
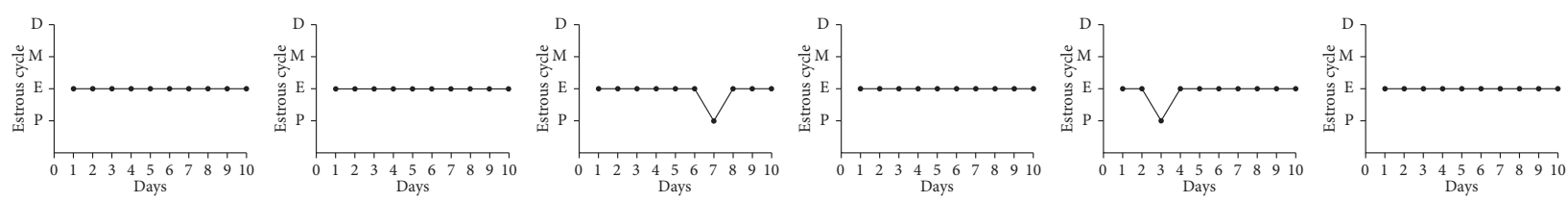

(f)

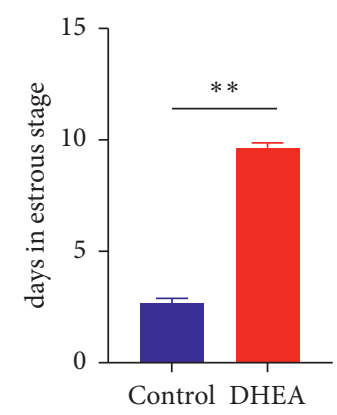

(g)

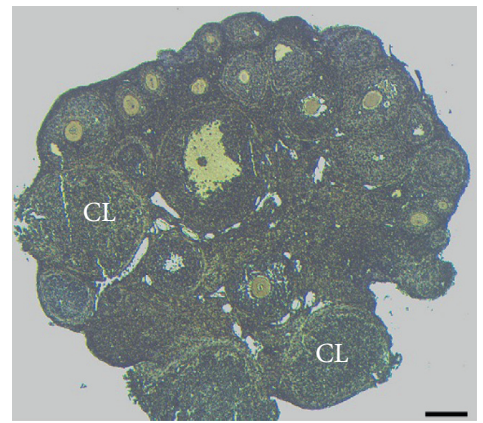

(j)

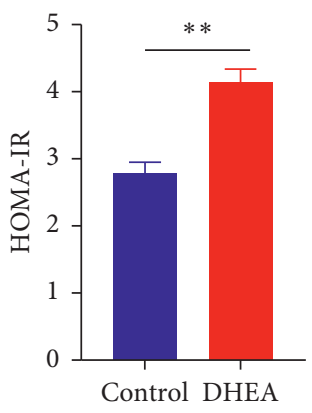

(h)

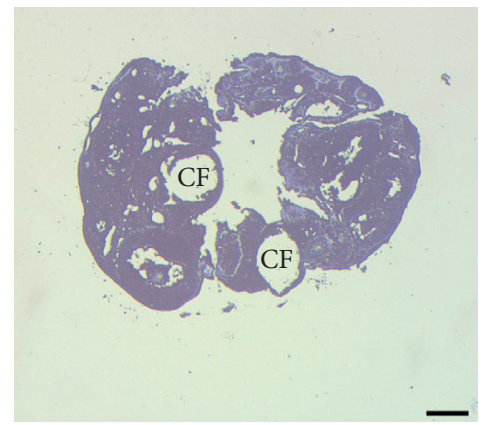

(k)

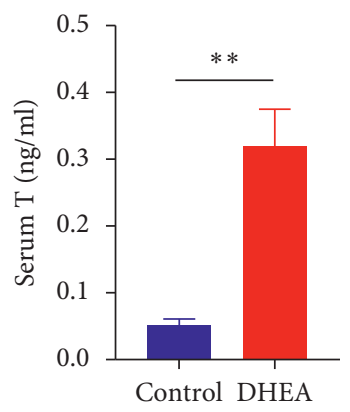

(i)

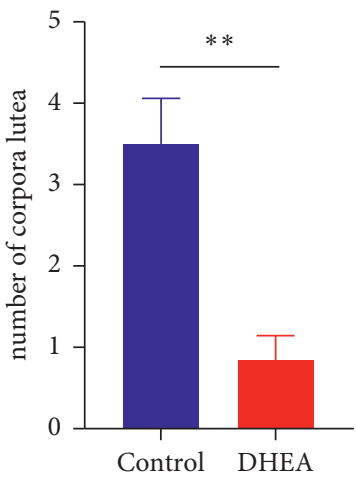

(l)

Figure 1: Continued. 


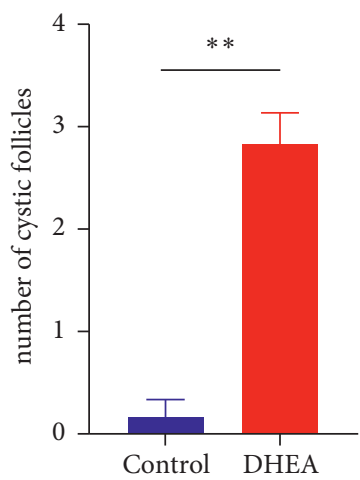

$(\mathrm{m})$

FIGURE 1: (a) Vaginal smears showing proestrus stage. (b) Vaginal smears showing estrus stage. (c) Vaginal smears showing metestrus stage. (d) Vaginal smears showing diestrus stage. Scale bar $=400 \mu \mathrm{m}$. (e) Estrous cycle of the control group. (f) Estrous cycle of the DHEA group. (g) Days in estrus stage. (h) HOMA-IR levels. (i) Serum T levels in mice. (j, k) Representative ovary morphologies from the control and DHEA groups. Scale bar $=200 \mu \mathrm{m}$. (l) The number of corpora lutea in ovaries. (m) The number of cystic follicles in ovaries. HOMA-IR: homeostasis model assessment-insulin resistance; T: testosterone; CL: corpus luteum; CF: cystic follicles. ${ }^{* *} p<0.01$.
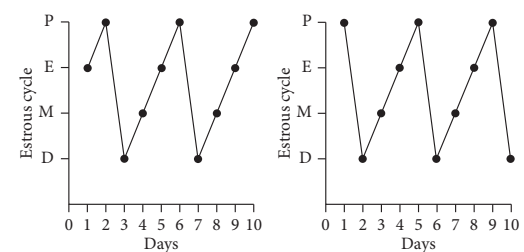

Days
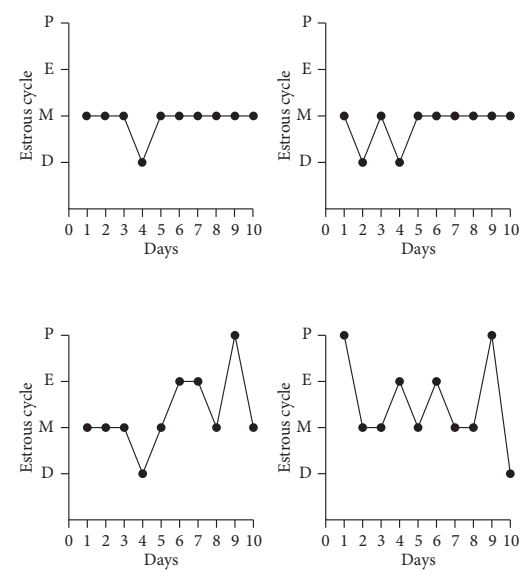

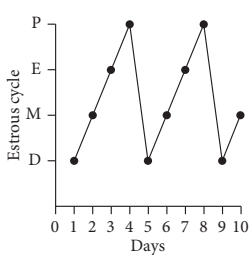

Days

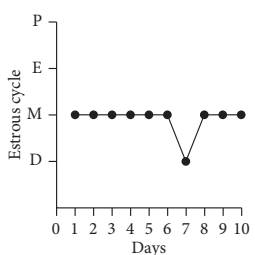

Days
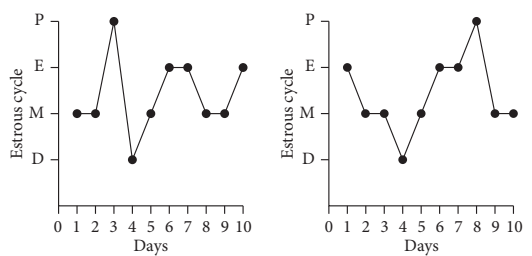

(c)

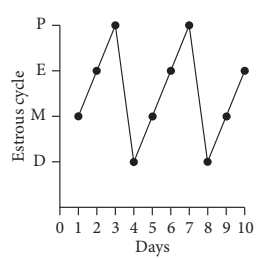

(a)

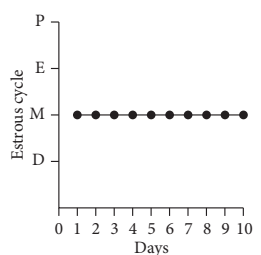

b)
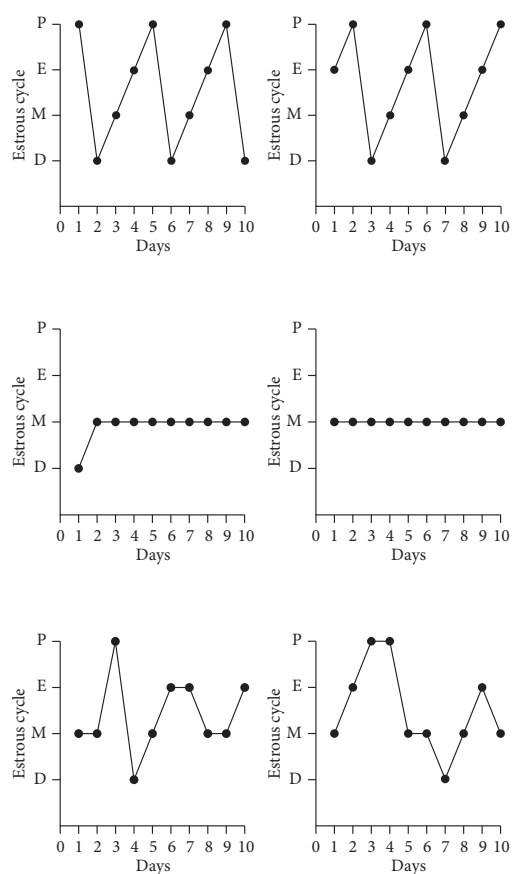

Figure 2: Continued. 


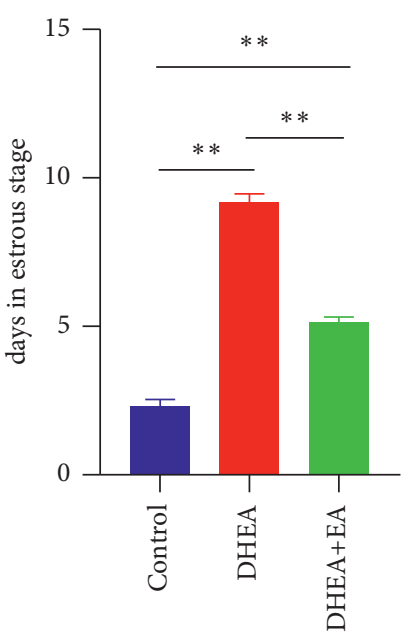

(d)

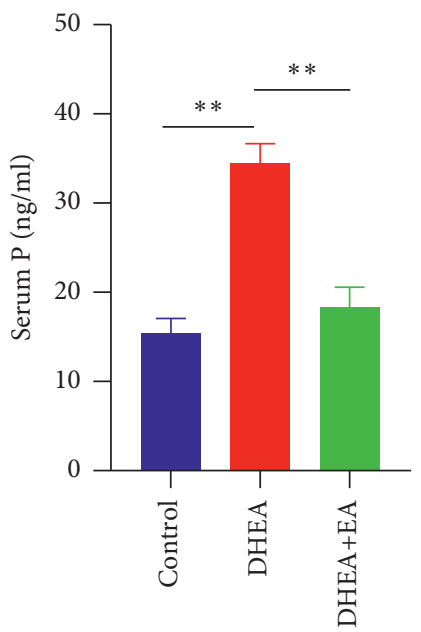

(g)

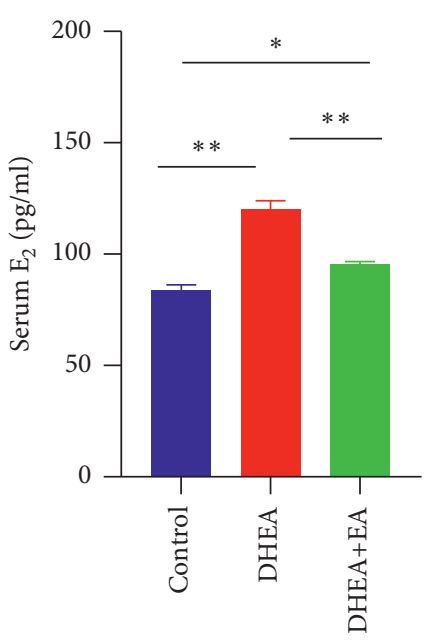

(e)

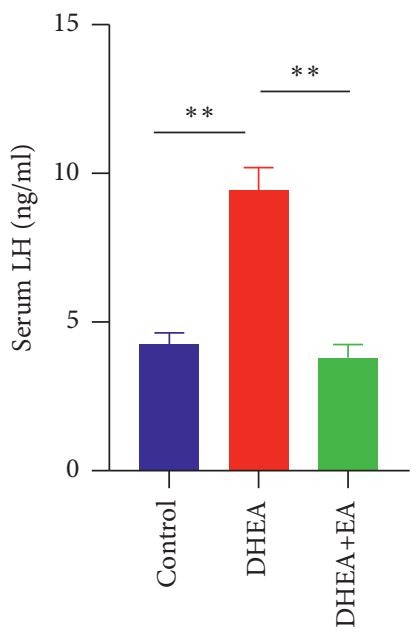

(h)

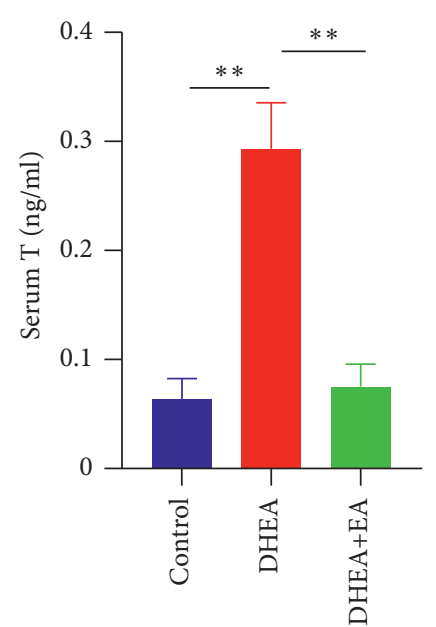

(f)

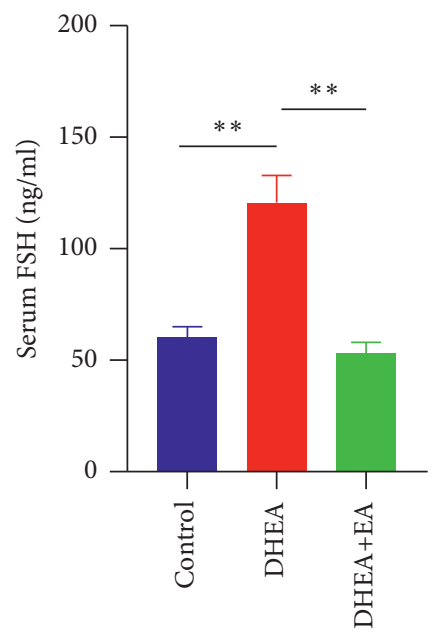

(i)

Figure 2: (a) Estrous cycle of the control group. (b) Estrous cycle of the DHEA group. (c) Estrous cycle of DHEA + EA group. (d) Days in estrus stage. (e-i) Serum $\mathrm{E}_{2}, \mathrm{~T}, \mathrm{P}, \mathrm{LH}$, and FSH levels in different groups of mice. $\mathrm{E}_{2}$ : estradiol, P: progesterone, T: testosterone, LH: luteinizing hormone, FSH: follicle-stimulating hormone. ${ }^{*} p<0.05$ and ${ }^{* *} p<0.01$.

expression of LHR to a normal level (Figure 3(c)). The average FSHR gene expression was about 2.5 times higher after DHEA treatment and was significantly decreased following EA treatment (Figure 3(d)). Thus, it can be seen that DHEA treatment significantly increased expression of hormone receptor genes in mouse ovaries, which may be an indirect cause of PCOS and which EA treatment can significantly improve. To determine the effects of EA on ovarian morphology, the ovaries of the three groups were stained with hematoxylin and eosin (Figures 3(e)$3(\mathrm{~g})$ ). We found that the number of CF was significantly decreased and there was an increasing trend in the number of CL in the DHEA+EA group compared to the DHEA group (Figures 3(h) and 3(i)). The data demonstrate that EA can ameliorate disordered follicular development.

\subsection{EA Improves Glucose Intolerance, Insulin Intolerance, and} HOMA-IR. To assess EA efficacy on glucose tolerance and insulin tolerance of the PCOS mice, IPGTT and IPITT were performed. After glucose injection, the blood glucose level of the
DHEA group was significantly higher than that of the control group at $30 \mathrm{~min}$ (Figure 4(a)). Mice in the DHEA + EA group had a similar blood glucose level at $30 \mathrm{~min}$ compared to the control group. Statistical analysis of IPGTT-AUC glucose levels showed a significant increase in the DHEA group mice compared to the control and DHEA + EA groups. There was no significant difference between the control group and the DHEA + EA group (Figure 4(b)). After insulin treatment, blood glucose levels in the DHEA mouse group at $30 \mathrm{~min}$ were higher compared to the control group and the DHEA + EA group, and levels did not return to normal by $120 \mathrm{~min}$ (Figure 4(c)). However, IPITTAUC among the three groups was not significantly different (Figure 4(d)). Additionally, fasting blood glucose, serum insulin level, and HOMA-IR were almost doubled in PCOS mice, an effect that was reversed by EA treatment (Figures 4(e)-4(f)).

\section{Discussion}

Because of the complexity of polycystic ovarian syndrome, there is no standardized treatment available; treatment is 


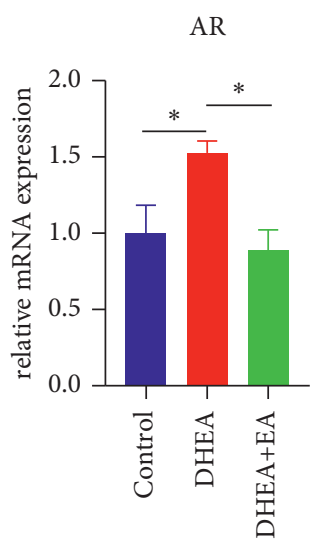

(a)

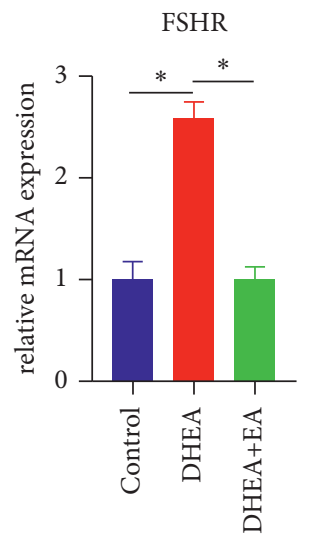

(d)

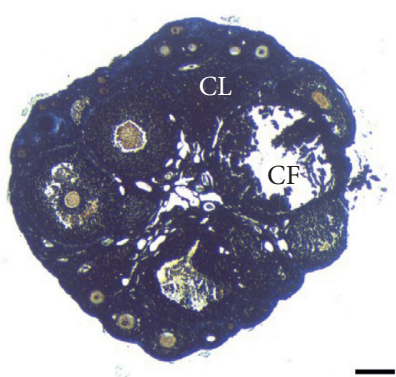

(g)

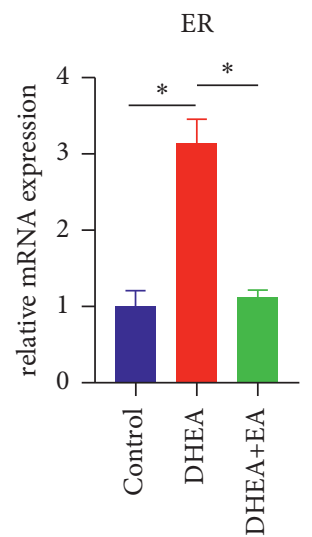

(b)

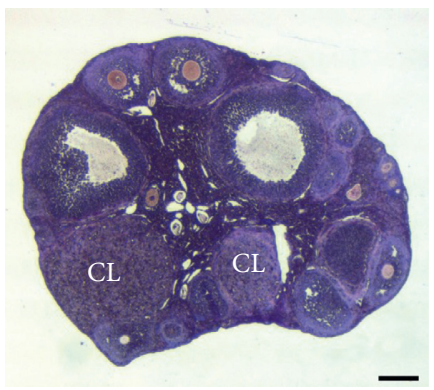

(e)

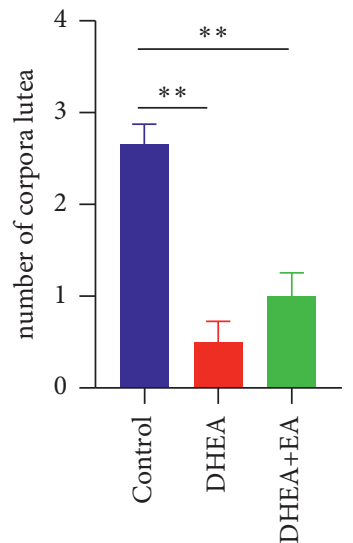

(h)

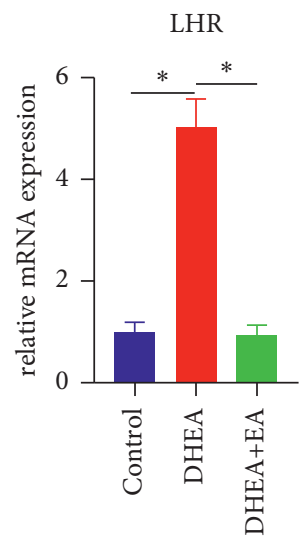

(c)

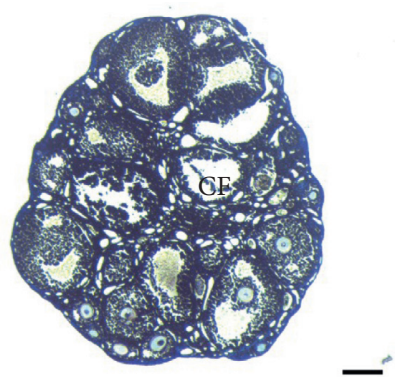

(f)

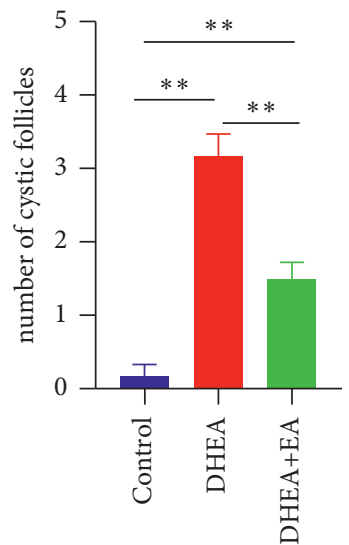

(i)

FIgUre 3: (a-d) AR, ER, LHR, and FSHR gene expressions in the ovaries. (e-g) Representative morphology from control group, DHEA group, and DHEA + EA group. Scale bar $=200 \mu \mathrm{m}$. (h) The number of corpus luteum cystic follicles in ovaries. (i) The number of cystic follicles in ovaries. AR: androgen receptor; ER: estrogen receptor; LHR: luteinizing hormone receptor; FSHR: follicle-stimulating hormone receptor. CF: cystic follicles, CL: corpus luteum. ${ }^{*} p<0.05$ and ${ }^{*} * p<0.01$.

individualized according to the symptoms and pathogenesis of each patient. For example, acne and hirsutism caused by excess androgen often have psychological repercussions in women with PCOS. Bleaching, plucking, shaving, waxing, chemical treatment, laser hair removal, and electrolysis could improve hirsutism, and dermabrasion, laser therapy, and light therapy can effectively treat acne [29-31]. With impaired glucose tolerance and insulin tolerance, women with PCOS are more prone to obesity, particularly central obesity. With PCOS development, increasing visceral fat deposition could induce high serum androgen production and reduced serum levels of sex-hormone-binding globulin 


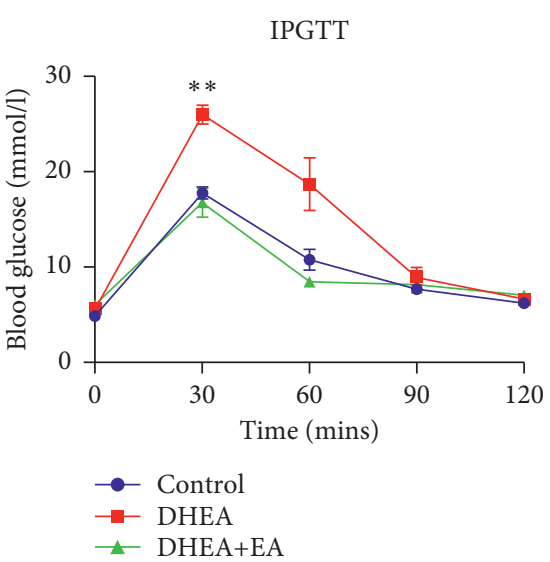

(a)

IPITT-AUC

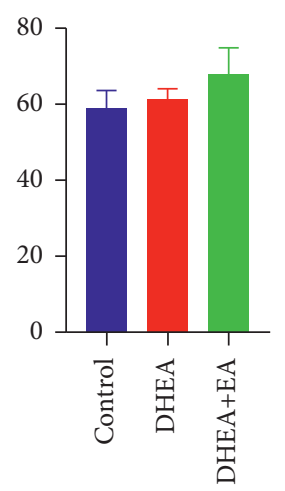

(d)
IPGTT-AUC

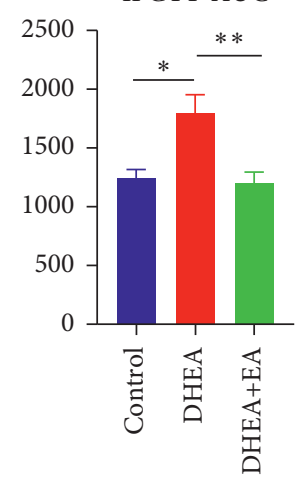

(b)

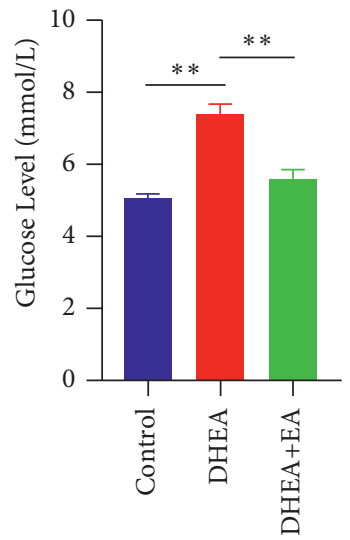

(e)

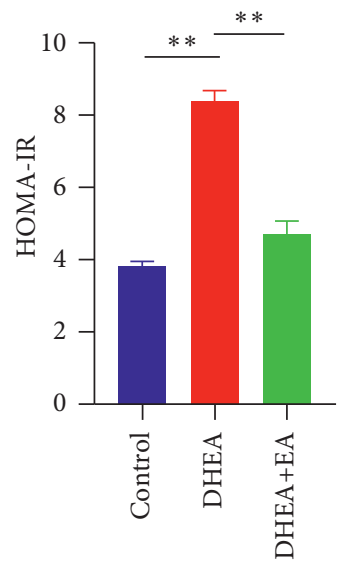

IPITT

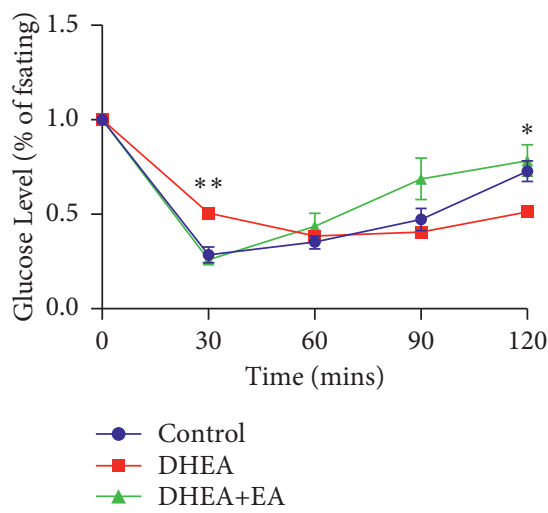

(c)

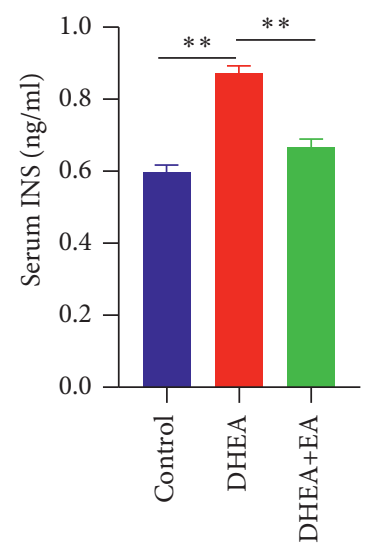

(f)

(g)

FIGURE 4: (a) IPGTT experiments. (b) AUC of glucose. (c) IPITT experiments. (d) AUC of glucose. (e) Fasting blood glucose. (f) Serum insulin levels. (g) HOMA-IR levels. IPGTT: intraperitoneal glucose tolerance test; AUC: area under the curve; IPITT: intraperitoneal insulin tolerance test; INS: insulin; HOMA-IR: homeostasis model assessment-insulin resistance. ${ }^{*} p<0.05$ and ${ }^{* *} p<0.01$.

[32]. Compared with normal women, obese women with PCOS are more likely to have metabolic abnormalities and severe cardiovascular conditions [33].

Oxidative stress has a pivotal impact on the biological functions of reproduction and metabolism and also plays a key role in the pathophysiology of PCOS. Excessive mitochondrial production of ROS can damage the function of mitochondria and even cause cell apoptosis. A previous study reported $\mathrm{CoQ} 10$ as an antioxidant compound could attenuate ovarian dysfunction induced by cyclophosphamide. CoQ10 reduced ROS levels and increased expression of the proliferation cell nuclear antigen (PCNA) and FSHR genes [34]. Another study reported that treatment with Panax ginseng extract $(100 \mu \mathrm{g} / \mathrm{mL})$ as 
an antioxidant not only had a positive effect on growth of isolated preantral follicles from the ovaries of prepubertal mice but also increased the expression of PCNA and FSHR genes [35]. Fariba Khodaeifar et al. revealed that treatment with Apium graveolens and Cinnamon zeylanicum could mitigate ovarian tissue injury in rats induced by PCOS. Serum levels of FBS, insulin, triglyceride, lowdensity lipoprotein, and cholesterol all showed obvious improvement [36].

Although the pathological mechanism of PCOS has not been clarified, most evidence has indicated that it has a close relationship with obesity and insulin resistance [37]. Considering the relations among IR, obesity, and cardiovascular disease, treating IR is a priority, especially in obese women with PCOS. Lifestyle modifications such as reasonable diet and increased exercise should be recommended to women with PCOS, especially those who also have IR or obesity. It is noteworthy that some studies have reported that oral antiobesity drugs resulted in initial positive changes in the signs and symptoms of PCOS; however, their long-term effectiveness remains unknown [38-40]. Bariatric surgery is a desirable treatment for women with IR and PCOS. A metaanalysis reported that this surgery could decrease weight and hirsutism in PCOS patients [41]. However, these therapies could not effectively improve IR symptoms, and they lack validation from long-term studies. Additional treatments for PCOS should therefore still be tested.

Acupuncture plays an essential role in traditional Chinese medicine, where it is widely accepted and used to treat many diseases, mainly because of the absence of side effects compared to many medicines. Electroacupuncture (EA) is a new treatment comprising traditional acupuncture combined with pulsed electrical stimulation. In recent years, the mechanism underlying the effect of EA has been widely studied. A randomized controlled trial found that, after 16 weeks of low-frequency EA treatment, body weight, body mass index, waist-hip ratio, oral glucose tolerance test, insulin release, and glucose and lipid metabolism indicators such as total cholesterol, triglycerides, and high-density lipoprotein cholesterol decreased compared with the baseline. The results show that EA improves insulin resistance in PCOS patients by regulating glucose and lipid metabolism [42]. The three acupoints used in this study are a common combination often used to treat metabolic diseases. After consulting the literature, we found that SP6 and ST36 are also very effective in the treatment of PCOS [22, 43, 44]. Furthermore, PC6 has the ability to clear meridians and regulate qi and xue in Chinese medicine, which is why we choose these three acupoints.

In this study, we successfully established a mouse model of PCOS with irregular estrous cyclicity; excessive estrogen, progesterone, and testosterone in serum; and increased plasma insulin, LH, and FSH levels. The pathological changes of ovaries were as follows: the number of corpora lutea and mature oocytes decreased, atresia follicles appeared, and expressions of AR, ER, PR, and GNRHR were increased. After EA treatment, excessive serum hormones and sex hormone receptors returned to normal levels, and ovarian morphology tended to improve. We also conducted
IPGTT and IPITT experiments, and the results suggested that, after EA treatment, mice with PCOS were more sensitive to glucose and insulin. Thus, EA had a positive effect on IR caused by PCOS.

The hypothalamus-pituitary-ovary axis regulates reproductive functions of female mice. In PCOS, the massive LH stimulated ovarian theca cells lead to production of more androgen. In ovaries, follicles are not sensitive to $\mathrm{FSH}$, which may be a critical factor in PCOS. This further causes reduced conversion efficiency of androgens to estrogens, leading to hyperandrogenism.

However, the relationship between IR and PCOS is unknown. Some studies have reported the mechanism of EA in the treatment of IR in PCOS. For instance, EA treatment could activate the activated protein kinase (AMPK) pathway in PCOS rats, inhibiting high expression of sterol regulatory element-binding protein-1 (SREBP1). Phosphorylation of insulin-stimulated insulin receptor $\beta$ and serine/threoninespecific protein kinase (AKT) returned to normal in primary granulosa cells [22].

Proopiomelanocortin (POMC) neurons in the arcuate nucleus of the hypothalamus (ARC) not only are a central regulator of energy homeostasis but also play a role in reproductive circuits. POMC neurons in mice with IR develop perturbations in maintenance of glucose metabolism and leptin sensitivity [45]. It is possible that EA improves IR by regulating POMC neurons. Research has found that $\mathrm{GnRH}$ neurons in the preoptic area express receptors for $\beta$-endorphin [46]. Furthermore, some POMC neurons in the ARC express Er $\alpha$ [47], and these neurons have been demonstrated to project to the preoptic area (POA), where GnRH neurons are located [48]. $\beta$-Endorphin in rats could inhibit GnRH and LH secretion [49]. $\alpha$-Melanocyte-stimulating hormone has an excitatory effect on the $\mathrm{GnRH}$ neuron by inducing central melanocortin receptors [50]. POMC neurons also regulate $\mathrm{GnRH}$ neurons by releasing $\gamma$-aminobutyric acid (GABA) and glutamate [51]. GABA is the main inhibitory neurotransmitter of the central nervous system. A previous study showed that mice with PCOS increased GABA innervations to $\mathrm{GnRH}$ neurons in the hypothalamus [52]. GABAB1 knockdown mice had a disordered estrus cycle and decrease in fecundity pulse frequency [53]. A meta-analysis suggested that valproate, a GABA-agonist drug, can be used to treat epilepsy, which has close associations with the development of PCOS. These results show that GABA neurons likely play an important role in gonadotropin hormones changes in PCOS [54]. It has also been suggested that EA could decrease $\beta$-endorphin concentration in both the hypothalamus and peripheral blood and increase ovarian blood and ovarian nerve growth factor (NGF) $[43,55,56]$. It is likely that EA recovers GNRH pulse by regulating $\beta$-endorphin and improving the concentration of hormones secreted by the ovaries.

In the present study, we demonstrated that EA has a considerable effect on DHEA-induced PCOS mice. However, our study did not address the mechanism of acupuncture in treating PCOS. We did not examine the effect of acupuncture on neuronal sensitivity to glucose and did not examine changes in insulin receptor and leptin receptor on 
POMC neurons. In future studies, we will focus on how EA treatment improves IR through POMC neurons.

\section{Conclusions}

In summary, our study suggests that EA has an ameliorative effect on PCOS model mice induced by DHEA. EA can improve estrous cycle, hormonal disorders, abnormal ovarian sex hormone receptors, ovarian morphology, and insulin resistance in PCOS mice. Therefore, EA may represent a valid therapeutic option for women with PCOS.

\section{Abbreviations}

EA: $\quad$ Electroacupuncture

DHEA: Dehydroepiandrosterone

AUC: Area under the curve

IPGTT: Intraperitoneal glucose tolerance test

IPITT: Intraperitoneal insulin tolerance test

LH: $\quad$ Luteinizing hormone

FSH: Follicle-stimulating hormone

HOMA: Homeostasis model assessment

IR: Insulin resistance

FSHR: Follicle-stimulating hormone receptor

LHR: Luteinizing hormone receptor

AR: Androgen receptor

ER: Estrogen receptor

GnRH: Gonadotropin-releasing hormone

AMPK: AMP-activated protein kinase

SREBP1: Sterol regulatory element-binding protein-1

AKT: $\quad$ Serine/threonine-specific protein kinase

POMC: Proopiomelanocortin

ARC: Arcuate nucleus of the hypothalamus

PCNA: Proliferation cell nuclear antigen

ROS: $\quad$ Reactive oxygen species.

\section{Data Availability}

Data used for analyses in this study are available from the corresponding author upon reasonable request.

\section{Ethical Approval}

This study was approved by the Ethical Committee of Guangzhou University of Chinese Medicine.

\section{Conflicts of Interest}

The authors declare that they have no conflicts of interest.

\section{Authors' Contributions}

Wei Yi and Shuai Cui designed the study. Chang Ji collected the data. Chang Ji, Wanling $\mathrm{Xu}$, and Zhiqing Zhang performed all experiments. Chang Ji and Wanling Xu wrote the paper. All authors read and approved the final manuscript. Chang Ji and Wanling Xu contributed equally to this work.

\section{Acknowledgments}

This study was supported by the National Natural Science Foundation of China (no. 81973946).

\section{References}

[1] Rotterdam ESHRE/ASRM-Sponsored PCOS Consensus Workshop Group, "Revised 2003 consensus on diagnostic criteria and long-term health risks related to polycystic ovary syndrome," Fertility and Sterility, vol. 81, no. 1, pp. 19-25, 2004.

[2] S. Sirmans and K. Pate, "Epidemiology, diagnosis, and management of polycystic ovary syndrome," Clinical Epidemiology, vol. 6, pp. 1-13, 2013.

[3] G. Conway, D. Dewailly, E. Diamanti-Kandarakis et al., "The polycystic ovary syndrome: a position statement from the European Society of Endocrinology," European Journal of Endocrinology, vol. 171, no. 4, pp. P1-P29, 2014.

[4] N. Ferri and M. Ruscica, "Proprotein convertase subtilisin/ kexin type 9 (PCSK9) and metabolic syndrome: insights on insulin resistance, inflammation, and atherogenic dyslipidemia," Endocrine, vol. 54, no. 3, pp. 588-601, 2016.

[5] B. C. J. M. Fauser, B. C. Tarlatzis, R. W. Rebar et al., "Consensus on women's health aspects of polycystic ovary syndrome (PCOS): the Amsterdam ESHRE/ASRM-Sponsored 3rd PCOS Consensus Workshop Group," Fertility and Sterility, vol. 97, no. 1, pp. 28-38, 2012.

[6] D. A. Dumesic, S. E. Oberfield, E. Stener-Victorin, J. C. Marshall, J. S. Laven, and R. S. Legro, "Scientific statement on the diagnostic criteria, epidemiology, pathophysiology, and molecular genetics of polycystic ovary syndrome," Endocrine Reviews, vol. 36, no. 5, pp. 487-525, 2015.

[7] C. Burt Solorzano, C. McCartney, S. Blank, K. Knudsen, and J. Marshall, "Hyperandrogenaemia in adolescent girls: origins of abnormal gonadotropin-releasing hormone secretion," BJOG: An International Journal of Obstetrics and Gynaecology, vol. 117, no. 2, pp. 143-149, 2010.

[8] S. H. Abtahi-Eivari, M. Moghimian, M. Soltani et al., "The effect of Galega officinalis on hormonal and metabolic profile in a rat model of polycystic ovary syndrome," International Journal of Womens Health and Reproduction Sciences, vol. 6, no. 3, pp. 276-282, 2018.

[9] F. Khodaeifar, A. Khaki, M. Torbati, and M. Shokoohi, "Investigating the role of hydroalcoholic extract of Apium graveolens and cinnamon zeylanicum on metabolically change and ovarian oxidative injury in a rat model of polycystic ovary syndrome," International Journal of Women's Health and Reproduction Sciences, vol. 7, no. 1, pp. 92-98, 2019.

[10] A. Dunaif, "Insulin resistance and the polycystic ovary syndrome: mechanism and implications for pathogenesis $*$," Endocrine Reviews, vol. 18, no. 6, pp. 774-800, 1997.

[11] E. Dahlgren, P. O. Janson, S. Johansson, L. Lapidus, G. Lindstedt, and L. Tengborn, "Hemostatic and metabolic variables in women with polycystic ovary syndrome** Supported by grants from the faculty of medicine, university of göteborg, the göteborg medical society, the research and development foundation, bohuslandstinget, and the hjalmar svensson fund," Fertility and Sterility, vol. 61, no. 3, pp. 455-460, 1994.

[12] S. Behboudi-Gandevani, F. Ramezani Tehrani, M. Rostami Dovom et al., "Insulin resistance in obesity and polycystic ovary syndrome: systematic review and meta-analysis of 
observational studies," Gynecological Endocrinology, vol. 32, no. 5, pp. 343-353, 2016.

[13] S. Behboudi-Gandevani, M. Amiri, R. Bidhendi Yarandi et al., "The risk of metabolic syndrome in polycystic ovary syndrome: a systematic review and meta-analysis," Clinical Endocrinology, vol. 88, no. 2, pp. 169-184, 2018.

[14] M. Rostami Dovom, F. Ramezani Tehrani, S. Djalalinia, L. Cheraghi, S. Behboudi Gandavani, and F. Azizi, "Menstrual cycle irregularity and metabolic disorders: a population-based prospective study," PloS One, vol. 11, no. 12, Article ID e0168402, 2016.

[15] D. P. Baldani, L. Skrgatic, R. Ougouag, and M. Kasum, “The cardiometabolic effect of current management of polycystic ovary syndrome: strategies of prevention and treatment," Gynecological Endocrinology, vol. 34, no. 2, pp. 87-91, 2018.

[16] J. Johansson and E. Stener-Victorin, "Polycystic ovary syndrome: effect and mechanisms of acupuncture for ovulation induction," Evidence-based Complementary and Alternative Medicine: eCAM, vol. 2013, Article ID 762615, 16 pages, 2013.

[17] E. Jedel, F. Labrie, A. Odén et al., "Impact of electro-acupuncture and physical exercise on hyperandrogenism and oligo/amenorrhea in women with polycystic ovary syndrome: a randomized controlled trial," American Journal of Physiology-Endocrinology and Metabolism, vol. 300, no. 1, pp. E37-E45, 2011.

[18] L. Yu, Y. Liao, H. Wu et al., "Effects of electroacupuncture and Chinese kidney-nourishing medicine on polycystic ovary syndrome in obese patients," Journal of Traditional Chinese Medicine, vol. 33, no. 3, pp. 287-293, 2013.

[19] Y. Cao, H. Chen, D. Zhao et al., "The efficacy of Tung's acupuncture for sex hormones in polycystic ovary syndrome: a randomized controlled trial," Complementary Therapies in Medicine, vol. 44, pp. 182-188, 2019.

[20] J. Johansson, L. Redman, P. P. Veldhuis et al., "Acupuncture for ovulation induction in polycystic ovary syndrome: a randomized controlled trial," American Journal of PhysiologyEndocrinology and Metabolism, vol. 304, no. 9, pp. E934E943, 2013.

[21] L. M. Pastore, C. D. Williams, J. Jenkins, and J. T. Patrie, "True and sham acupuncture produced similar frequency of ovulation and improved LH to FSH ratios in women with polycystic ovary syndrome," Journal of Clinical Endocrinology \& Metabolism, vol. 96, no. 10, pp. 3143-3150, 2011.

[22] Y. Peng, X. Yang, X. Luo et al., "Novel mechanisms underlying anti-polycystic ovary like syndrome effects of electroacupuncture in rats: suppressing SREBP1 to mitigate insulin resistance, mitochondrial dysfunction and oxidative stress," Biological Research, vol. 53, no. 1, p. 50, 2020.

[23] P. Cui, T. Ma, A. Tamadon et al., "Hypothalamic DNA methylation in rats with dihydrotestosterone-induced polycystic ovary syndrome: effects of low-frequency electro-acupuncture," Experimental Physiology, vol. 103, no. 12, pp. 1618-1632, 2018.

[24] L. Dou, Y. Zheng, L. Li et al., "The effect of cinnamon on polycystic ovary syndrome in a mouse model," Reproductive Biology and Endocrinology, vol. 16, no. 1, p. 99, 2018.

[25] E. Anderson, M.-T. Lee, and G. Y. Lee, "Cystogenesis of the ovarian antral follicle of the rat: ultrastructural changes and hormonal profile following the administration of dehydroepiandrosterone," The Anatomical Record, vol. 234, no. 3, pp. 359-382, 1992.

[26] G. Y. Lee, J. M. Croop, and E. Anderson, "Multidrug resistance gene expression correlates with progesterone production in dehydroepiandrosterone-lnduced polycystic and equine chorionic gonadotropin-stimulated ovaries of prepubertal Rats1," Biology of Reproduction, vol. 58, no. 2, pp. 330-337, 1998.

[27] C. G. Luchetti, M. E. Solano, V. Sander et al., "Effects of dehydroepiandrosterone on ovarian cystogenesis and immune function," Journal of Reproductive Immunology, vol. 64, no. 1-2, pp. 59-74, 2004.

[28] E. C. Ndeingang, P. B. Defo Deeh, P. Watcho, and A Kamanyi, "Phyllanthus muellerianus (euphorbiaceae) restores ovarian functions in letrozole-induced polycystic ovarian syndrome in rats," Evidence-based Complementary and Alternative Medicine: eCAM, vol. 2019, Article ID 2965821, 16 pages, 2019.

[29] H. F. Escobar-Morreale, E. Carmina, D. Dewailly et al., "Epidemiology, diagnosis and management of hirsutism: a consensus statement by the androgen excess and polycystic ovary syndrome society," Human Reproduction Update, vol. 18, no. 2, pp. 146-170, 2012.

[30] S. J. Kempiak and N. Uebelhoer, "Superficial chemical peels and microdermabrasion for acne vulgaris," Seminars in $\mathrm{Cu}$ taneous Medicine and Surgery, vol. 27, no. 3, pp. 212-220, 2008.

[31] M. H. Gold, D. J. Goldberg, and M. S. Nestor, "Current treatments of acne: medications, lights, lasers, and a novel 650- $\mu$ s 1064-nm Nd: YAG laser," Journal of Cosmetic Dermatology, vol. 16, no. 3, pp. 303-318, 2017.

[32] D. S. Kiddy, P. S. Sharp, D. M. White et al., "Differences in clinical and endocrine features between obese and non-obese subjects with polycystic ovary syndrome: an analysis of 263 consecutive cases," Clinical Endocrinology, vol. 32, no. 2, pp. 213-220, 1990.

[33] B. Yildirim, N. Sabir, and B. Kaleli, "Relation of intra-abdominal fat distribution to metabolic disorders in nonobese patients with polycystic ovary syndrome," Fertility and Sterility, vol. 79, no. 6, pp. 1358-1364, 2003.

[34] A. Delkhosh, M. Delashoub, A. A. Tehrani et al., "Upregulation of FSHR and PCNA by administration of coenzyme Q10 on cyclophosphamide-induced premature ovarian failure in a mouse model," Journal of Biochemical and Molecular Toxicology, vol. 33, no. 11, Article ID e22398, 2019.

[35] A. Majdi Seghinsara, H. Shoorei, M. M. Hassanzadeh Taheri et al., "Panax ginseng extract improves follicular development after mouse preantral follicle 3D culture," Cell Journal, vol. 21, no. 2, pp. 210-219, 2019.

[36] F. Khodaeifar, S. M. B. Fazljou, A. Khaki et al., "Investigating the role of hydroalcoholic extract of Apium graveolens and Cinnamon zeylanicum on metabolically change and ovarian oxidative injury in a rat model of polycystic ovary syndrome," International Journal of Women's Health and Reproduction Sciences, vol. 7, no. 1, pp. 92-98, 2019.

[37] U. A. Ndefo, A. Eaton, and M. R. Green, "Polycystic ovary syndrome: a review of treatment options with a focus on pharmacological approaches," $P$ and T: A Peer-Reviewed Journal for Formulary Management, vol. 38, no. 6, pp. 336355, 2013.

[38] L. Cho, E. Kilpatrick, B. Keevil, A. Coady, and S. Atkin, "Effect of metformin, orlistat and pioglitazone treatment on mean insulin resistance and its biological variability in polycystic ovary syndrome," Clinical Endocrinology, vol. 70, pp. 233$237,2008$.

[39] V. Jayagopal, E. S. Kilpatrick, S. Holding, P. E. Jennings, and S. L. Atkin, "Orlistat is as beneficial as metformin in the treatment of polycystic ovarian syndrome," Journal of Clinical Endocrinology \& Metabolism, vol. 90, no. 2, pp. 729-733, 2005. 
[40] D. Panidis, K. Tziomalos, E. Papadakis, C. Vosnakis, P. Chatzis, and I. Katsikis, "Lifestyle intervention and antiobesity therapies in the polycystic ovary syndrome: impact on metabolism and fertility," Endocrine, vol. 44, no. 3, pp. 583-590, 2013.

[41] H. F. Escobar-Morreale, E. Santacruz, M. Luque-Ramírez, and J. I. Botella Carretero, "Prevalence of 'obesity-associated gonadal dysfunction' in severely obese men and women and its resolution after bariatric surgery: a systematic review and meta-analysis," Human Reproduction Update, vol. 23, no. 4, pp. 390-408, 2017.

[42] H. X. Dong, Q. Wang, Z. Wang et al., "Impact of low frequency electro-acupuncture on glucose and lipid metabolism in unmarried PCOS women: a randomized controlled trial," Chinese Journal of Integrative Medicine, vol. 3, no. 6, pp. 158-161, 2021.

[43] U. R. Budihastuti, E. Melinawati, S. Sulistyowati, and I. Nurwati, "Electroacupuncture effect on polycystic ovary syndrome to improve oocytes' growth," Medical Acupuncture, vol. 31, no. 6, pp. 379-383, 2019.

[44] X. Tong, Y. Liu, X. Xu et al., "Ovarian innervation coupling with vascularity: the role of electro-acupuncture in follicular maturation in a rat model of polycystic ovary syndrome," Frontiers in Physiology, vol. 11, p. 474, 2020.

[45] Q. Tang, Y. Gao, Q. Liu et al., "Sirt6 in pro-opiomelanocortin neurons controls energy metabolism by modulating leptin signaling," Molecular Metabolism, vol. 37, Article ID 100994, 2020.

[46] A. H. Lagrange, O. K. Rønnekleiv, and M. J. Kelly, "Estradiol17 beta and mu-opioid peptides rapidly hyperpolarize $\mathrm{GnRH}$ neurons: a cellular mechanism of negative feedback?" Endocrinology, vol. 136, no. 5, pp. 2341-2344, 1995.

[47] Y. Xu, T. P. Nedungadi, L. Zhu et al., "Distinct hypothalamic neurons mediate estrogenic effects on energy homeostasis and reproduction," Cell Metabolism, vol. 29, no. 5, p. 1232, 2019.

[48] S. X. Simonian, D. P. Spratt, and A. E. Herbison, "Identification and characterization of estrogen receptor?-Containing neurons projecting to the vicinity of the gonadotropin-releasing hormone perikarya in the rostral preoptic area of the rat," The Journal of Comparative Neurology, vol. 411, no. 2, pp. 346-358, 1999.

[49] S. P. Kalra and P. S. Kalra, "Nutritional infertility: the role of the interconnected hypothalamic neuropeptide Y-galaninopioid network," Frontiers in Neuroendocrinology, vol. 17, no. 4, pp. 371-401, 1996.

[50] K. Backholer, J. Smith, and I. J. Clarke, "Melanocortins may stimulate reproduction by activating orexin neurons in the dorsomedial hypothalamus and kisspeptin neurons in the preoptic area of the Ewe," Endocrinology, vol. 150, no. 12, pp. 5488-5497, 2009.

[51] S. T. Hentges, V. Otero-Corchon, R. L. Pennock, C. M. King, and M. J. Low, "Proopiomelanocortin expression in both GABA and glutamate neurons," Journal of Neuroscience, vol. 29, no. 43, pp. 13684-13690, 2009.

[52] A. M. Moore, M. Prescott, C. J. Marshall, S. H. Yip, and R. E. Campbell, "Enhancement of a robust arcuate GABAergic input to gonadotropin-releasing hormone neurons in a model of polycystic ovarian syndrome," Proceedings of the National Academy of Sciences, vol. 112, no. 2, pp. 596-601, 2015.

[53] P. N. Catalano, N. Di Giorgio, M. M. Bonaventura, B. Bettler, C. Libertun, and V. A. Lux-Lantos, "Lack of functional GABAB receptors alters GnRH physiology and sexual dimorphic expression of GnRH and GAD-67 in the brain,"
American Journal of Physiology-Endocrinology and Metabolism, vol. 298, no. 3, pp. E683-E696, 2010.

[54] X. Hu, J. Wang, W. Dong, Q. Fang, L. Hu, and C. Liu, "A meta-analysis of polycystic ovary syndrome in women taking valproate for epilepsy," Epilepsy Research, vol. 97, no. 1-2, pp. 73-82, 2011.

[55] E. Stener-Victorin and C. Lindholm, "Immunity and $\beta$-endorphin concentrations in hypothalamus and plasma in rats with steroid-induced polycystic ovaries: effect of low-frequency Electroacupuncture1," Biology of Reproduction, vol. 70, no. 2, pp. 329-333, 2004.

[56] L. Mannerås, S. Cajander, M. Lönn, and E. Stener-Victorin, "Acupuncture and exercise restore adipose tissue expression of sympathetic markers and improve ovarian morphology in rats with dihydrotestosterone-induced PCOS," American Journal of Physiology - Regulatory, Integrative and Comparative Physiology, vol. 296, no. 4, pp. R1124-R1131, 2009. 